\title{
Erratum to: Pairwise interaction of drops in shear-thinning inelastic fluids
}

\section{Sadegh Mohammadi Masiri, Morteza Bayareh* and Afshin Ahmadi Nadooshan \\ Department of Mechanical Engineering, Shahrekord University, Shahrekord, Iran}

Erratum to: Korea-Australia Rheology Journal, 31(1), 25-34 (2019).

DOI: $10.1007 / \mathrm{s} 13367-019-0003-8$

Due to an error at the publisher, Eq. (1) for authors Sadegh Mohammadi Masiri et al. was listed incorrectly. The original Eq. (1) is as follows:

$\frac{\partial(\rho \boldsymbol{u})}{\partial t}+\nabla \cdot(\rho \boldsymbol{u} \boldsymbol{u})=-\nabla p+\nabla \cdot \boldsymbol{\tau}+\sigma \int \kappa \boldsymbol{n} \delta^{2}\left(\boldsymbol{x}-\boldsymbol{x}^{\prime}\right) d s$ 